\title{
Brûler les champs, pour voir ensuite ce qui va repousser
}

\section{Éléonore Pitre et Frannie Holder}

Frannie Holder est une compositrice, chanteuse, et multi-instrumentiste de Montréal. Membre des groupes pop-hip hop Random Recipe et électro-folk Dear Criminals, l'artiste polyvalente a débuté sa carrière musicale en 2007. Axé sur la performance live, Random Recipe a rapidement été amené à jouer à l'international. Parallèlement, son groupe Dear Criminals s'inscrit dans une démarche de création multidisciplinaire et cumule les collaborations avec le milieu théâtral (Les Lettres d'Amour-Québec/Rouen), la danse contemporaine (TRAPPED, Things are leaving quietly in silence, Suites Ténébreuses), l'opéra (SeX'Y_Opéra de Paris), les arts numériques (STEREOSCOPIC—Québec/Berlin) et le cinéma (Fatale Station, POMPÉl, $N E L L Y$ ). La musique composée pour le long-métrage NELLY leur a d'ailleurs valu l'IRIS de la meilleure musique originale au Gala Québec Cinéma en 2017. Durant la pandémie, ils ont élaboré le spectacle LONE RIDE présenté au Lion d'Or à Montréal, puis au Cabaret de la dernière chance à Rouyn-Noranda, et bientôt au Pantoum à Québec. Frannie Holder a également composé la musique pour le court métrage de Marianne Farley et la musique d'un documentaire de Patrick Bossé.

Éléonore Pitre est une guitariste accompagnatrice qui a grandi dans le milieu de musique traditionnelle de la région de Lanaudière. Elle est une des membres fondatrices du groupe folk Rosier qui se démarque surtout à l'international (Australie, Danemark, Royaume-Uni, NouvelleZélande, États-Unis). Elle a accompagné plusieurs artistes pour différentes tou rnées ou spectacles de variétés sur la scène québécoise au cours des dernières années (Spectacle "Une chance qu'on s'a », spectacle hommage à Plamondon «Stone », tournée de René Simard, etc.). Durant la pandémie, le groupe Rosier a terminé leur nouvel album Légèrement qui est sorti en janvier 2021. Éléonore Pitre joue présentement dans l'ensemble de l'émission télévisuelle Star Académie.

Cette conversation a eu lieu le 22 juillet 2020.

Éléonore Pitre : Comment les annonces du 13 mars t'ont affectée professionnellement?

Frannie Holder : J'avais quatre mois de tournée devant moi. Un premier mois, en mars, en Italie avec Random Recipe et au mois d'avril, avec Dear Criminals, j'allais en France. Je devais retourner en Italie avec Random Recipe en juin et au mois d'août on s'en allait dans l'Ouest canadien. C'était 80 spectacles au total. C'est certain que tout ça s'est écroulé et que financièrement c'est une grande perte, puisque ce sont les mois qui sont les plus lucratifs de l'année. En fait, c'est le cumule des cachets de spectacles et des contrats de composition pour de la musique de film qui font mon année financière.

Mais en même temps, je t'avoue que moi ça ne m'a pas trop dérangée au final, parce que je flirtais encore avec mon « burn out » du mois d'octobre. J'étais un peu au repos et au ralenti depuis. Je n'ai eu qu'une seule tournée en Amérique latine au mois de décembre, qui au final avait été assez relax, puisque plusieurs shows ont été annulés en raison des manifestations qui se déroulaient là-bas. Si je suis bien honnête, quand on a annulé la tournée en Italie, j'étais soulagée de ne pas avoir à faire ma valise et de reprendre l'avion. C'est drôle à dire, parce que dans le fond, c'est tout ce que je veux : réussir, faire de la tournée et avoir du job, mais quand tu te rends compte qu'il arrive des affaires comme la pandémie et que tu n'es même pas déçue, ça te fait réaliser qu'il y a beaucoup de sacrifices à cette job-là. Tu sais bien ce que c'est, la tournée : ne pas être dans tes affaires, l'impact sur tes relations personnelles et intimes, 
l'impact environnemental ...

Je pense que je ne me permettais pas de reconnaître que c'était un effort et que j'y allais de reculons parce que c'est exceptionnel notre métier et c'est toujours ce que j'ai voulu faire. Par contre, au final, quand tu te rends compte que tu es soulagée de ne pas partir, ça en dit beaucoup.

Et toi?

EP : De mon côté, avec Rosier, je partais en Australie à la fin de mars et pour moi, c'était un peu comme un retour à la vie de tournée, ce que j'aime le plus faire. Après une année plus sédentaire où mon band a fait beaucoup de « rebranding » en restant au pays, et où j'ai davantage enseigné, c'était vraiment excitant pour moi de retourner en Australie, un pays où j'ai vécu mes plus belles expériences de spectacle.

Au départ, c'était un peu la panique et ça m'a rendue triste de ne pas partir, en plus de penser aux revenus perdus, mais ce qui m'a fait relativiser, c'est de me rendre compte que tout le monde à travers la planète était dans la même situation. J'étais persuadée que tout allait arrêter pour une période indéterminée, jusqu'à 2021 et plus ! Je me suis mise à pratiquer tous les jours, chose que je n'ai jamais le temps de faire quand je suis sur la route. Je profitais du temps que j'avais tout à coup.

Finalement, j'ai eu la chance de me faire appeler à participer à un show télé comme musicienne pigiste, en plein milieu du confinement ! J'étais super surprise. C'est Alex McMahon (Louis-Jean Cormier, Daniel Bélanger, Ariane Moffatt, Plaster) qui m'a offert une place dans le « house band » pour le spectacle Une chance qu'on s'a à TVA. Un gros show qui rassemblait plusieurs artistes québécois de renom, pour faire une levée de fonds pour des organismes qui venaient en aide aux personnes âgées et aux femmes victimes de violence conjugale. Ça m'a donné un " high », j'étais en extase de pouvoir jouer de la musique sur une scène avec des artistes et musiciens que j'admire. L'expérience était très différente qu'à l'habitude : toute l'équipe de tournage portait des masques et des gants, tout le monde se tenait à deux mètres et les artistes passaient les uns après les autres. II n'y avait pas de rassemblement festif auquel on est habitué après un spectacle; c'était chacun de son bord. C'était une expérience vraiment spéciale. Ç'a été dur après de retourner me confiner dans mon petit appartement après avoir eu la chance et le plaisir de rejouer sur une scène quand peu de gens pouvaient le faire. La pandémie a vraiment été une pause obligatoire pour tous et il y a quelque chose que j'ai trouvé vraiment intéressant là-dedans. Tout le monde se remet en question, se demande quoi faire, se demande s'il se plaisait dans ce qu'il faisait avant, se demande quel effet positif ça peut avoir sur sa vie. On pense également aux bienfaits écologiques d'une pause mondiale.

FH : Ben oui! La musique qu'on fait, les spectacles qu'on fait, le pourquoi on le fait. Ça va tellement vite que comment tu consommes de la musique, ce que tu fais sur scène, ce que tu composes, ça devient un peu une gibelotte. J'en suis venue à ne même plus aller voir de show et j'ai arrêté d'écouter de la musique, car tu en vois tellement, il y en a tellement, il y a tellement d'offres, que ça devient du « bruit » pour moi. Comme créateur, tu crées, tu crées, tu crées, après ça tu pars en tournée, puis ensuite c'est déjà le temps de créer le prochain projet. II n'y a jamais un moment où tu t'arrêtes, tu expires et que tu vois où les choses se déposent en toi, que tu analyses comment tu les as vécues et ensuite tu inspires : « what's next? ».

EP : Souvent, le «what's next? » vient tout de suite—des fois, avant même d'avoir terminé ce sur quoi tu travailles dans le moment. 
FH : Oui! Puis, il n'y a jamais de moment pour décompresser, pour juste vivre et « processer » ce que tu viens d'expérimenter, et l'échange que tu as eu avec le public. Ça devient unisens, et pour moi, ça, ça n'a aucun sens. T'sais, on met des choses dans l'univers, on crée des choses, des moments de grâce, des chansons qui accompagnent le monde, pour avoir un échange avec les gens. De cette façon, on les accompagne dans leur quotidien, on fait partie d'une réflexion globale, etc. Alors, quand nous-mêmes, qui sommes là pour offrir une trame sonore à leur vie, on n'a jamais de moment de pause-qu'est-ce qu'on crée ? Qu'est-ce qu'on a à dire ? Qu'est-ce qu'on crée comme trame sonore, comme espace où les gens sont supposés pouvoir fuir, se déposer, vivre toutes sortes d'émotions?

Ce n'est pas pour rien que je n'écoute plus de musique et que je ne vais plus voir de spectacle. À un moment donné, ça devient juste des trames de fond et de la distraction. L'arrêt pour moi, ça a été d'être toute seule à la maison pendant trois mois en silence et on dirait que je laissais treize ans de travail se déposer en moi. Et puis ça m'a permis de me poser des questions comme: "Qu'est-ce que j'ai aimé faire? », «Qu'est-ce que je n'ai pas aimé faire? », "Qu'estce que j'ai envie de faire pour la suite? ». L'image que j'avais, c'était de bruler les champs, pour ensuite voir ce qui va repousser. De mon point de vue, je regardais comment l'industrie fonctionne; on est toujours dans la mentalité qu'il ne faut pas perdre le « momentum ». Qui a le luxe de prendre une année sabbatique dans le milieu de la musique? Sauf que dans tout ça, quand tu es créateur, tu es supposé avoir un espace où tu ressens des choses, un temps d'introspection, et puis par la suite, tu les sors. Sinon qu'est-ce que tu dis? Tu dis du vide, quelque chose de compressé, de pas réfléchis, de la surface, et c'est le gros problème de notre industrie selon moi: le vide qu'on raconte et le peu d'espace qu'on a pour dire des choses qui sont vraies et qui font écho à la société pour plus que huit secondes et quart.

EP: Je crois justement que la pandémie et l'arrêt forcé pour tous vont peut-être avoir donné aux artistes et au public un temps de réflexion à savoir ce qu'ils recherchent quand ils créent/consomment de la musique. Avant, on était habitué à un train de vie qui allait super vite et peut-être que certains ne se posaient même pas de question sur ce qu'ils écoutaient. Ils ouvraient la radio et se disaient probablement : c'est ce qui m'est offert, alors c'est ce que je consomme. De mon côté, j'ai eu beaucoup de questionnements à ce sujet durant la pandémie : quelle musique ou quels artistes est-ce que j'ai envie d'encourager, quels artistes m'inspirent vraiment, qu'est-ce que moi j'ai envie de véhiculer comme message quand je suis sur une scène, etc. Avec l'énorme «boom » de «perfo live » sur Facebook qu'on a vu apparaitre dans les premiers mois du confinement, c'était vraiment un drôle de moment d'abondance de contenu (gratuit en plus); ça débordait de partout, tous les soirs. C'était un peu étourdissant selon moi.

Quelle a été ta réponse face aux changements précipités de la pandémie sur notre métier ? L'annulation de spectacles, de tournées, l'impact des exigences de la distanciation sociale, le fameux virage numérique?

FH: Moi quand tout ça est arrivé, le confinement, la fermeture de tout, j'étais vraiment contente, je me disais : «pandémie forever ! » (rires). Mais je n'avais pas envie de créer. J'avais juste envie d'expirer, de profiter d'avoir du temps pour moi. T'sais quand tu es parti à l'étranger six mois par année, le moment où tu es à Montréal, tu te sens coupable de prendre du temps pour toi, tu dois voir tes amis, ta famille, etc. Là, je me suis vraiment permis de prendre le temps et de voir ce qui allait se passer dans mon cœur, dans ma tête, dans ma vie, dans mes ambitions. Puis, vraiment tranquillement, j'avais des idées qui me venaient, mais je me retenais de ne pas aller en studio, d'exécuter l'idée tout de suite comme je l'aurais fait auparavant. Je me demandais si c'était un réflexe de productivité, ou bien si enfin c'était ma réelle inspiration qui 
revenait?

J'étais vraiment prudente avec tout ce qui se présentait en moi, à savoir si c'était juste par habitude de toujours être active ou si j'en avais vraiment envie.

EP: Mais j'ai vu que tu t'es remise à la musique live, avec Dear Criminals! Vous avez conçu un spectacle génial avec tout le contexte d'isolement et de distanciation sociale, non ? Comment l'idée de Lone Ride vous est venue?

FH: C'est une idée qu'on avait eue il y a quelques années. On avait fait un show où on était dans un cube avec un public autour de nous à 360 degrés. On s'était dit que ça serait drôle d'inverser le concept: de jouer pour une personne à la fois, avec nous qui l'entourons sur scène. On n'avait jamais vraiment réussi à trouver comment le concept pourrait fonctionner, comment ça pourrait être possible financièrement pour que ce soit rentable. Quand est arrivée la pandémie, Vincent (guitariste dans le groupe) nous a écrit un courriel en relançant l'idée et en disant que c'était le moment de faire ce fameux spectacle pour une personne. J'étais tellement énervée quand j'ai lu ce courriel! Ça faisait des années que je n'avais pas été excitée pour un projet comme ça! Ça m'a fait du bien, ça faisait un mois et demi qu'on était en pause, donc ça m'avait laissé le temps de redevenir enthousiaste. Pendant quatre jours au Lion d'Or, on a fait des marathons: on jouait une chanson par personne et c'était celle de leur choix. On jouait environ sept heures par jour. La personne était assise sur scène, face à la salle et on était autour d'elle, derrière des Plexiglas.

\section{EP: Comment as-tu vécu l'expérience?}

FH: Je n'ai jamais rien vécu de tel dans ma vie et j'en ai fait beaucoup de shows. Ça fait treize ans que je fais ça et c'est le spectacle qui m'a le plus rentré dedans de ma vie. $90 \%$ du monde pleurait. Pour plusieurs d'entre eux, c'était leur première sortie depuis trois mois, certains n'avaient vu personne depuis trois mois. La détresse et la solitude des gens m'ont vraiment touchée et marquée. Ça m'a fait réaliser qu'un public habituel, ce n'est pas juste plein de gens qui ont acheté un billet, mais bien plein d'individus qui ont une histoire précise et bien à eux. Ce spectacle-là va, je l'espère, me faire voir les prochains publics différemment d'avant. C'était de faire de la musique complètement autrement, comme je n'ai jamais fait avant. On a eu une couverture médiatique comme jamais auparavant, parce qu'il ne se passait rien d'autre en même temps. Ç'a été une bonne façon pour le Lion d'Or de faire parler de la salle et des enjeux qui se dressent pour les salles de spectacle au Québec en temps de pandémie. On a refait la même formule à Rouyn-Noranda, grâce au FME (Festival de Musique Émergente). On s'est tous rendus là-bas, chacun dans notre voiture. On ne pensait vraiment pas refaire de la tournée cet été!

Avec Rosier, vous venez d'enregistrer un album. Qu'est-ce que vous pensez faire pour la sortie?

EP: C'est sûr qu'avec tous ces changements, il faut s'improviser de nouvelles tactiques pour interagir avec les gens, de musicien à spectateur/auditeur. On est en train de réfléchir plus que jamais à une idée intéressante et originale pour faire un lancement d'album. On prend le temps de bien réfléchir, et une chose est sûre : les gens ont envie d'avoir de nouvelles choses à écouter et il y a de la place pour sortir de la nouvelle musique, beaucoup plus que pour faire de la musique live! Donc, on veut bien faire ça. En attendant la sortie de l'album, on sort des singles en vidéoclip plus que jamais. On a dû se « revirer sur un dix cents » pour notre single qui est sorti en début juin, car on voulait tourner un clip, mais c'était en plein confinement alors 
c'était impossible. Béatrix, la chanteuse et violoniste du band, a dû faire un vidéo complètement « fait maison » avec des vidéos souvenirs de notre dernière tournée, captés avec nos iPhones. On a eu de la chance par ailleurs que notre dernière tournée avant la pandémie soit en Nouvelle-Zélande - ça a donné de belles images! On a fait ce qu'on pouvait avec ce qu'on avait. On a deux autres vidéoclips qui devraient sortir d'ici l'automne. C'est très différent de ce qu'on a l'habitude de vivre, car je dirais que $80 \%$ des spectacles qu'on fait durant une année sont hors Québec. Présentement, rien n'est possible à l'international, donc c'est du jamais vu pour le band ce qu'on vit présentement.

FH: Je trouve important de souligner aussi comment cette mise en pause a affecté tous les musiciens pigistes et les techniciens qui travaillent sur les spectacles habituellement. Les programmes d'aides du gouvernement ont été dirigés vers les créateurs artistiques, alors ça laisse beaucoup de gens de côté qui font partie de l'écosystème du milieu de la musique. Avec Random Recipe, on a décidé de donner un cachet symbolique à nos collègues musiciens et techniciens qui travaillent avec nous habituellement et qui sont peut-être en train de se dire qu'ils vont changer de carrières parce que les bourses et les demandes de subventions ne les visent pas directement. J'ai vu ce genre de solidarité dans le milieu chez plein de gens qui sont indépendants, comme nous.

EP: Oui, c'est très cool de voir la solidarité et l'entraide qui sont ressorties chez les gens. Malheureusement, le nombre de musiciens pigistes et de techniciens qui ont eu la chance d'avoir du travail depuis le mois de mars est très bas.

Et puis as-tu recommencé à écouter de la musique finalement ?

FH: Ça m'a pris du temps! J'ai recommencé, mais juste des vinyles, des objets qui ont une continuité, qui ont été créés de $A$ à $Z$ dans un esprit et un mode de pensée précis. II y a un rituel, et j'ai l'impression d'être dans le respect de ce que l'artiste a voulu raconter à ce momentlà. Je me suis même mise à danser dans mon salon! Je me disais " yes », je recommence à avoir un contact purement émotif et enfantin avec la musique. 